\title{
ELECTRICAL ACTIVITY OF SINGLE INTERNODE
}

\author{
KIKUJI KONISHI* \\ Department of Physiology, Tokyo Medical and Dental University, Tokyo
}

The saltatory conduction theory for myelinated fibres was criticized recently by Rosenblueth et al. (1954). They showed that the membrane current accompanying conduction alternates not only at the nodes, but also at every point along internodal stretches (continuous conduction theory). Yamagiwa (1951) expressed years ago a view that the internodal part may be excited, but perhaps later by successive stimuli from proximal as well as distal nodes, to be left behind to die out there, contributing nothing to normal impulse transmission. If the internode is really an excitable structure as they claim or suppose, then it should respond also to artificial stimuli. The present work was undertaken to confirm this point, examining the internodal membrane current in details. Results obtained with single internode preparations support the views of Rosenblueth et al. and Yamagiwa in the sense that the internode is excitable.

\section{METHOD}

Materials and experimental method were essentially the same as those used previously (Konishi, 1957). Particular points are as follows:

(1) The pool in the middle (about $0.8 \mathrm{~mm}$. wide) contained only a part of an internodal segment, the nodes being located in the larger, lateral pools.

(2) Applying a stimulus (a long square pulse current, cathodal or anodal) to an internode, or stimulating the trunk properly, examinations were made on the internodal membrane currents directly evoked or accompanying conduction.

(3) For current recording, a D.C. amplifier of low resistance input and a Braun tube oscilloscope were employed.

In all records presented in this paper, the upward deflection represents positivity at the grid electrode, if not particularly mentioned. Temperature ranged $9.5-29.0^{\circ} \mathrm{C}$. The results obtained were satisfactorily reproducible.

$$
\text { RESULTS }
$$

Direct Stimulation of Internode

\section{Evidences for internodal "response"}

Typical examples of records obtained are presented in fig. 1. The left two, taken as controls, show the current across an internodal membrane in normal conditions. The familiar double-peaked shape proves the right location of the middle pool on an internode. Records on the right were obtained by passing

Received for publication June 16, 1958.

* 小西喜久治 

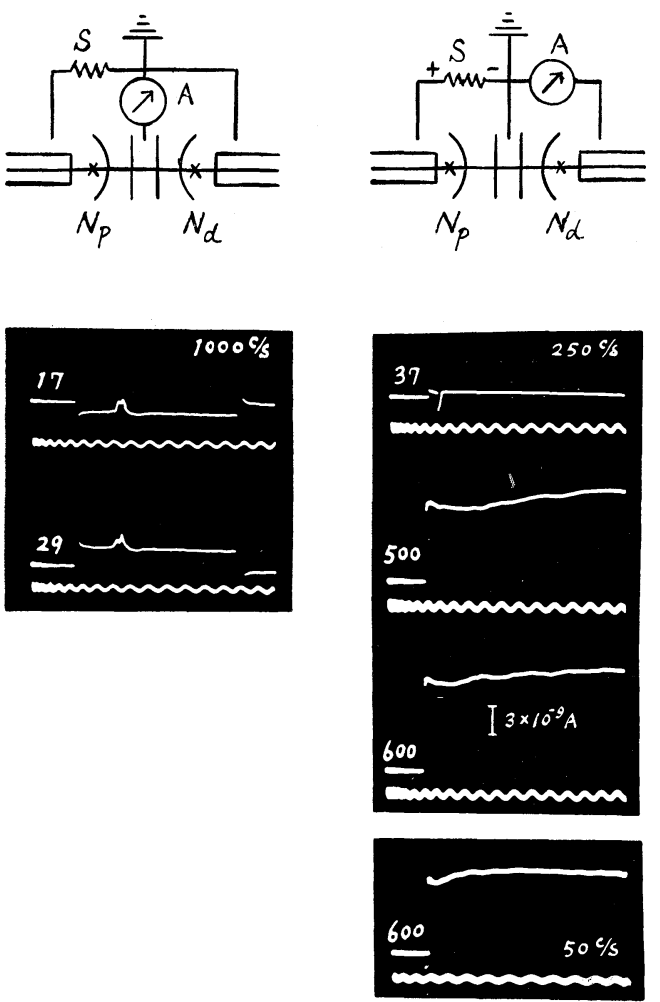

a long outward pulse through the internodal membrane. A weak current brought forth nothing particular except the spike generated by Nd (right, top). With stronger stimuli, however, there appeared a long upward deflection following the spike (right, except the top). We take this as a sign of "make response" of the internode.

FIG. 1. Internodal responses elicited by a long outward current (right, except the top). All three pools filled with normal Ringer. No node of Ranvier present in the middle pool. $S$ denotes the output of a square pulse generator, and $A$ the current recording apparatus. Left two records taken as controls, to show the membrane current caused by activities of $N_{p}$ and $N_{a}$. Strengths of stimulating pulse given on the left, in $\mathrm{mV}$. Time marks, $1,000,250$ and $50 \mathrm{c} / \mathrm{s}$. Temperature $21.0^{\circ} \mathrm{C}$. Toad's fibre, $16 \mu$ thick. Diagram on the right applies to figs. 2-6.

It might be argued that the recorded deflections are not referable to any active process, but only to passive, impedance changes taking place at the nodal and/or internodal membranes. There are, however, several evidences for the active nature, among others the following three:

(a) Responses evokable by cathodic current only. The make response was readily and repeatedly elicitable with an outward current pulse of a duration longer than $0.5 \mathrm{msec}$. (compare Fig. 2, $a, b$ with $c$ ), but never with an inward.

(b) Survival of the "response". The "response" could survive for a certain period after the break of the current (fig. 2). In the figure, a hypertonic Nacitrate solution was applied beforehand to the internode under study, in order to lower the threshold (see the next section, a). We see there clearly that the "response" survives, and that regularly longer with increasing size of the response (the longest duration obtained was about $4 \mathrm{msec}$.). It might be possible that the internodal membrane polarized by the stimulating current discharges through the measuring circuit after the break, in the same direction as in the make. If so, the survival is only apparent, but it will be highly improbable that the discharging current lasts so long as mentioned above. The survival of activity is, on the other hand, a fact well established in many excitable cells. 

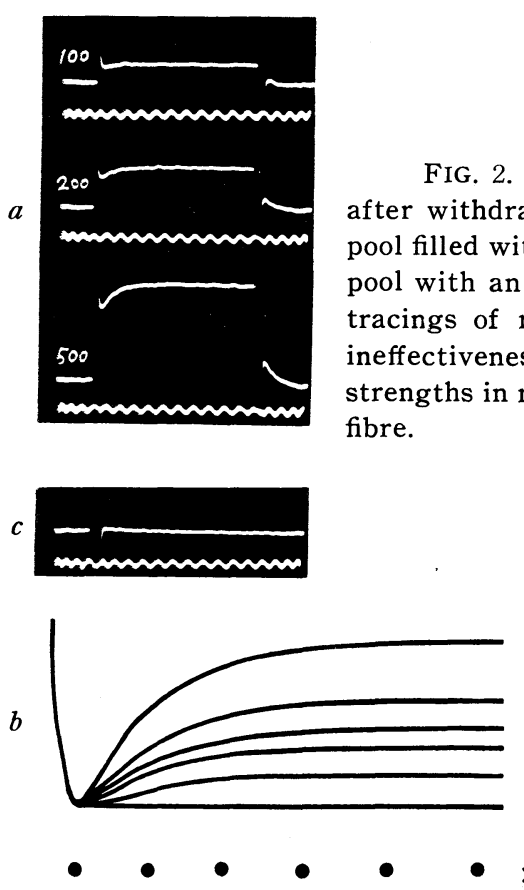

- msec.
FIG. 2. Survival of internodal responses after withdrawal of applied pulse. The middle pool filled with a $6 \% \mathrm{Na}$-citrate and the distal pool with an isotonic $\mathrm{KCl}$ solution. Record $b$, tracings of records $a$ 's. Record $c$ shows the ineffectiveness of a short pulse $(2.0 \mathrm{~V})$. Pulse strengths in $\mathrm{mV} .1,000 \mathrm{c} / \mathrm{s} .13 .0^{\circ} \mathrm{C} .13 \mu$ thick fibre. (c) Anode break excitation. Under similar arrangements and conditions as above, we applied to the internode anodal current pulses of a constant duration, expecting to have break excitations, which are readily elicitable in single nodes of Ranvier (Konishi, 1957; Ichioka and Konishi, 1957). As seen in Fig. 3, the pulse was followed, again, by an upward excursion after the break. It is particularly note-worthy that the excursion started after the complete subsidence of the counter current, which is the stimulus for break excitation. This supplies an unquestionable evidence for the active nature of the excursion.

Opening responses thus obtained were generally graded in duration as well as in size. The duration was sometimes so long as about 40 msec., but sometimes so short as usual nodal spikes (fig. 4, left). Similar responses could be evoked also by cathodal stimulation of an internode subjected to anelectrotonus (fig. 4, right).
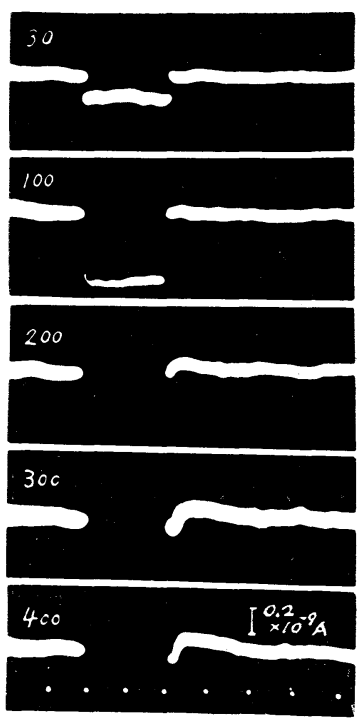

FIG. 3. Anode break responses of an internode. The middle pool filled with a $5 \%$ $\mathrm{NaCl}$ and the distal pool with a 3\% urethane Ringer solution. Strengths of the anodal pulse given in $\mathrm{mV}$. $50 \mathrm{c} / \mathrm{s}$. $24.0^{\circ}$ C. $11 \mu$ thick fibre. 

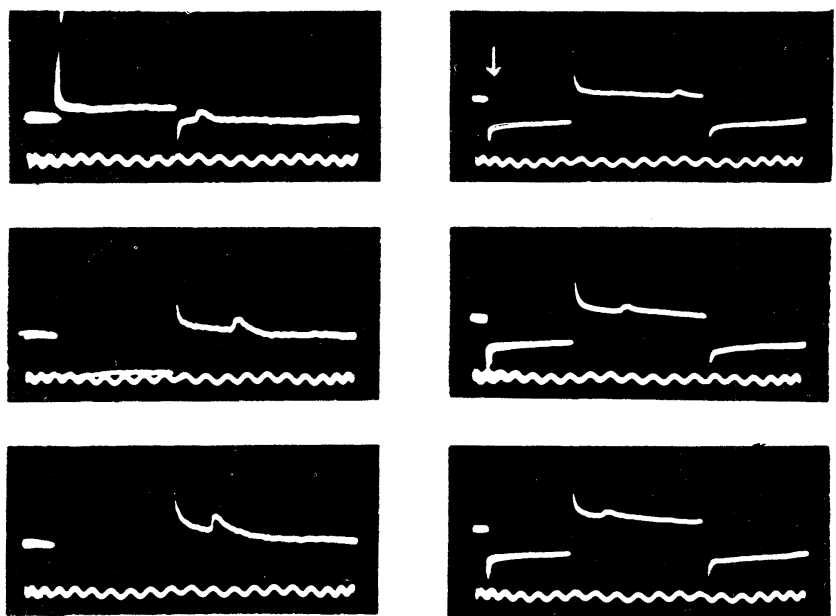

FIG. 4. Short responses of an internode. The middle pool filled with a $5 \% \mathrm{NaCl}$ and the distal pool with an isotonic $\mathrm{KCl}$ solution. Left: top, anode break response of the node $\mathrm{Np}$, taken as control; lower two, break responses of the internode obtained after reversing the polarity of the pulse. Pulse strengths, from above, 50, 200 and $400 \mathrm{mV}$. Right: cathode make responses of the internode subjected to $300 \mathrm{mV}$ anelectrotonus. The beginning of the polarizing current indicated by arrow. Strengths of stimulating pulse, from above, 300 , 350 and $400 \mathrm{mV}$. Amplification lowered on the right. $250 \mathrm{c} / \mathrm{s} .9 .5^{\circ} \mathrm{C}$. $10 \mu$ thick fibre.

\section{Other features of the response (vice-evidences for the activity)}

(a) Threshold and its variation. A certain definite threshold existed in each preparation. The rheobasic voltage was normally around $450 \mathrm{mV}$, that is, about 15 times that for nodal excitation. The ineffectiveness of short pulses (fig. 2,c) seems to be mainly due to such a high threshold.

Isotonic solutions of pure sucrose or electrolytes such as $\mathrm{NaCl}, \mathrm{KCl}$ and Na-citrate, brought about little or no significant change in threshold. Of a particular interest was the action of hypertonic (5\%) $\mathrm{NaCl}$ and hypertonic (6\%) sodium citrate solutions, which lowered the threshold and favored the occurrence of break excitation (figs. 2, 3 and 4). Narcotics were almost effectless, but saponine, a myelin solvent, eliminated the excitability soon after application.

The threshold was lowered by cathodal, while raised by anodal, polarization of sufficient strengths $(150-350 \mathrm{mV})$ and duration $(30 \mathrm{msec}$.), as in other excitable tissues.

(b) Strength-response time-relation. The response time for long pulses of threshold intensity was usually several $10 \mathrm{msec}$. The relation between the response time and the pulse strength fits well with Weiss's formula (fig. 5).

(c) Size, duration and shape of the response. The size of the response varied with stimulus intensity. The maximal one obtained was comparable with that of the nodal spike $\left(2-3 \times 10^{-9} \mathrm{~A}\right.$ ) (figs. 1, 4 and 6). A striking difference from the nodal response lies in the duration. As stated before (fig. 3), 


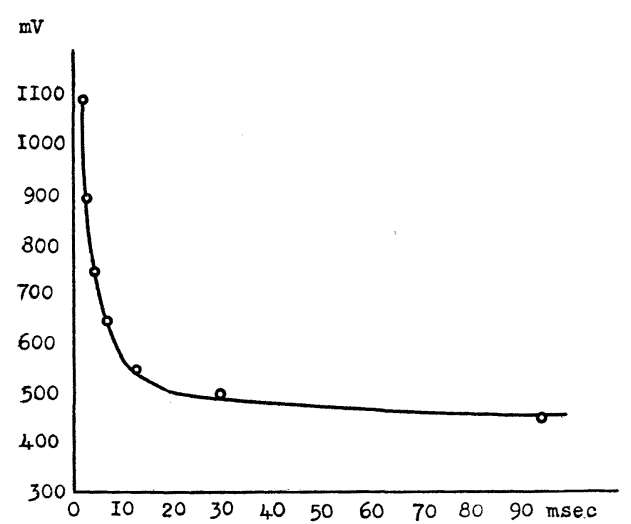

FIG. 5. Relation between the pulse strength (ordinate) and the latency of internodal make response (abscissae), obtained with a $13 \mu$ thick fibre at $22.0^{\circ} \mathrm{C}$. The continuous curve drawn according to Weiss's formula $v=a+b / t$, where $a$ is $446 \mathrm{mV}$ and $b$ $1,269 \mathrm{mV} \cdot \mathrm{msec}$. the duration of the break response was variable while the make response outlasted the whole period of stimulation or longer. A possible mechanism will be stated in CONSIDERATION.

Responses were in general of a simple shape, composed of a more or less long rising phase followed by a falling phase with (make response) or without (break response) a maintained plateau between them. The rising phase of the make responses showed not infrequently an irregular or a steplike variation in weak stimulations (figs. 1 and 6, right). It is very important to note that the response became in no case diphasic during the whole period of activity (figs. 1-4 and 6).

\section{Relation between internodal and nodal activities}

Make responses were often accompanied by repetitive spikes of the distal node, $N_{d}$ (fig. 6, left). They were, however, perhaps resulted from the spread of the stimulating current, and have nothing to do with the internodal response. For confirming this, the same examination was made after application of a $3 \%$ Na-citrate solution to the node to fire it repetitively (fig. 6, right). Inspite of the activity being developed at the internode, there appeared no appreciable sign of influence imposed upon the spike pattern. The enhancement of the spike frequency and the depression of the height are evidently due to catelectrotonic effects of the stimulating current, as in the left records.

\section{Responses from the juxta-nodal region \\ The responses described so far have been obtained from a central}
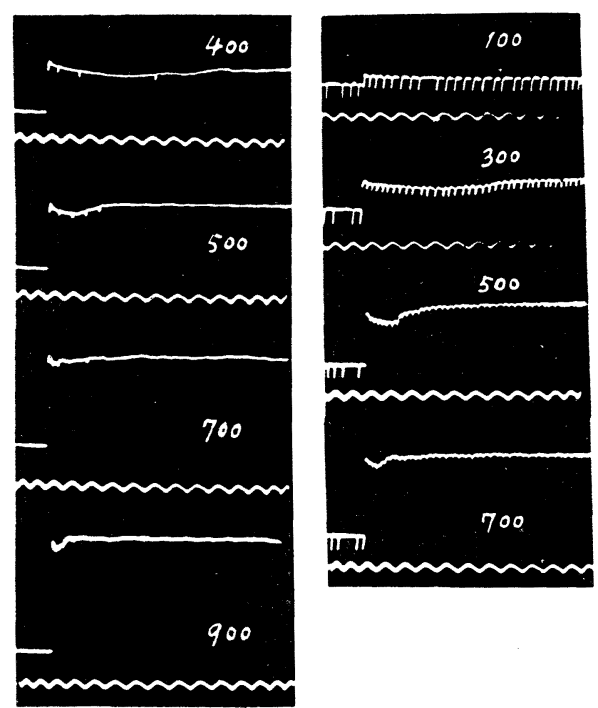

FIG. 6. Internodal response and repetitive spikes of the distal node in companion. In experiments on the right half, a $3 \%$ Na-citrate solution was introduced into the distal pool. $50 \mathrm{c} / \mathrm{s}$. $13 \mu$ thick fibre. $22.0^{\circ} \mathrm{C}$. 
portion of an internode separated by air-gaps from adjacent nodes of Ranvier. Similar responses were obtainable also from the periferal, viz., juxtanodal portions of an internode. The method employed was the same with that for single node experiments (Konishi, 1957). A node of Ranvier, $N_{m}$, was introduced into the middle pool of Ringer, while two others, $N_{p}$ and $N_{d}$, in the lateral pools containing narcotica (fig. 7). Under such an arrangement, the stimulating current will be led into the nodal as well as juxta-nodal portions, and accordingly it may give rise to a juxta-nodal response, provided that the stimulus is intense enough. This expectation was confirmed in an experiment as follows:

Stimulating pulses weaker than $500 \mathrm{mV}$ yielded only a single or repetitive spikes originating from $N_{m}$ (fig. 7, left column). With stronger stimuli, however, the spike disappeared (perhaps became invisible) completely and, instead, new upward deflections were materialized (middle column).

The new response thus obtained was similar with the internodal in several essential features, i.e., it was (a) inelicitable by anodal current (right column), (b) unmodifiable by narcotization, (c) graded in size, the maximal one attaining nearly that of a full-sized nodal spike, and (d) elicitable even at the moment of maximal activity of $N_{m}$. All these facts do indicate that the response in question originates not in the node but in the adjacent myelinated portions.

The differences between the juxta-nodal and internodal responses are: (a) The rising phase of the internodal responses is often of an irregular or steplike form, as stated before, while that of the juxta-nodal responses always of a smooth, continuous shape. (b) In the case of internode, the plateau was maintained throughout the course of the stimulating current, while it was soon curtailed by a falling phase in the case of juxta-node. The reasons for these differences could not be clarified. Presumably, some structural differences, for example, in myelin lamellae formation, might play a role in some way or other 
for the first fact; we had also an experience suggesting that the curtailing of the juxta-nodal response might be due to activity of a distal juxta-node, but not quite clear.

\section{Internodal Membrane Current Accompanying Conduction}

\section{Moment of appearance of the inward component}

A reference was made previously to the internodal membrane current evoked by two adjacent nodes activated in succession as in normal conduction (fig. 1, left). It is very important to note here that the double-peaked outward current is always followed by an inward. This inward current was first analyzed by Huxley and Stämpfli (1949), and was regarded as a passive discharging current which, originating from the polarized internodal membrane, manifests itself with the abrupt termination of the source action potential. If so, the inward current should follow the termination of the action potential temporarily. This point was subjected to direct tests.

With the experimental arrangements shown diagrammatically in fig. 8, $a$, left, simultaneous records were taken of the internodal membrane current and the mono-nodal action current. The record shows clearly that the inward component of the former (upper trace) starts early in the falling phase, that is, distinctly before the end of the latter (lower trace). The point is that the mono-nodal action current thus recorded might be somewhat prolonged in duration by the passive discharging current which, originating from the polarized internodal membrane, adds itself to the genuine action current. To examine this effect, we took records of the mono-nodal action currents with and without a current contribution from the internodal part, for comparison (fig. 8, b). There was generally a little difference in spike height, but no significant difference in duration. It is certain therefore that the inward current in question appears well before the termination of the action current, accordingly, of the action potential, at least practically. The slight tendency of prolongation of duration in fig. $8, b$ (top) should rather be taken as showing a weak activity of the internode.
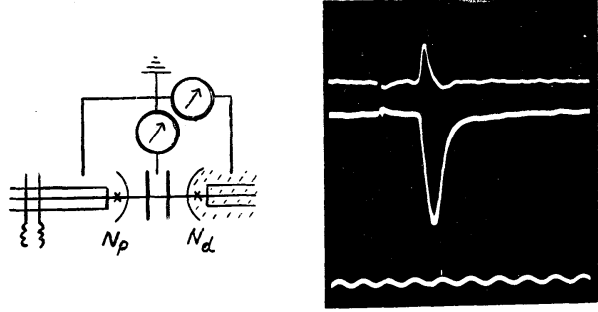
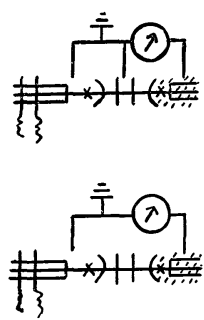

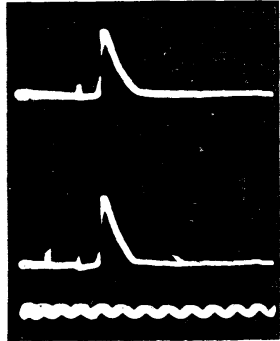

$b$

FIG. 8. $a$ : Simultaneous record of an internodal membrane current (upper trace) and a mono-nodal action current (lower trace). Distal nodes narcotized. 1,000 c/s. $14 \mu$ thick fibre. $26.5^{\circ} \mathrm{C}$. $b$ : Comparison of mono-nodal action currents obtained with an internode connected and disconnected with the input of the amplifier. $500 \mathrm{c} / \mathrm{s}$. $15 \mu$ thick fibre. $18.0^{\circ} \mathrm{C}$. 


\section{Size and duration of the inward current}

The inward current has been said to be just small by some authors (Stämpfli, 1954; Tasaki, 1955). In literatures, however, several records of very large amplitudes are found, for example, fig. 2 in Tasaki and Ushiyama (1950), fig. 5 in Sato and Schneider (1954) and fig. 2, $B$ in Maruhashi et al. (1956).

As far as examined, the most effective factor which influences the amplitude, seems to be the temperature. Data obtained from 22 preparations at various room temperatures are collected in fig. 9, where the amplitude is expressed in percentage of the peak value of the outward current. There is a considerable variation at each given temperature, presumably due in part to variation in fibre diameter, but the whole result shows unquestionably a tendency of the amplitude to become larger with higher temperature. Notice that, at temperatures higher than $19.5^{\circ} \mathrm{C}$., the relative amplitude averaged from 15 examples

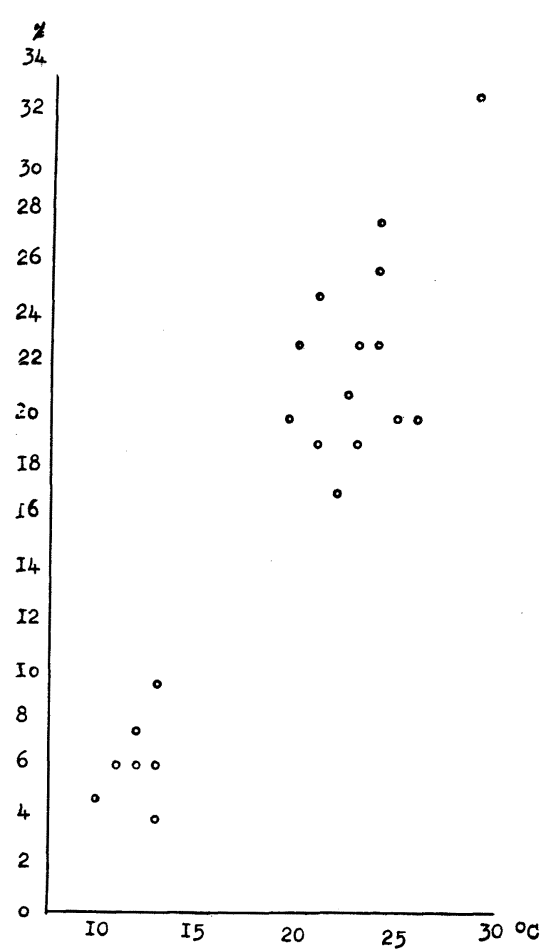

FIG. 9
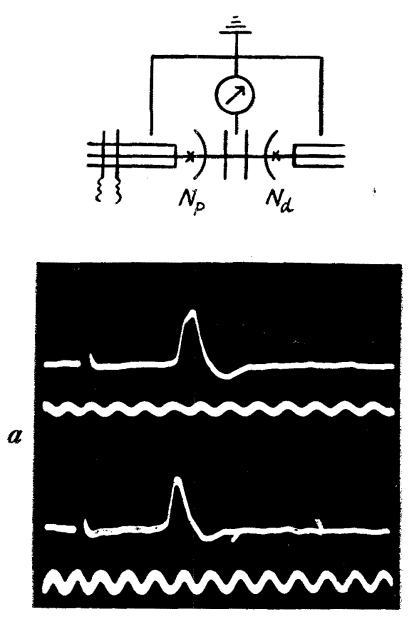

$b$

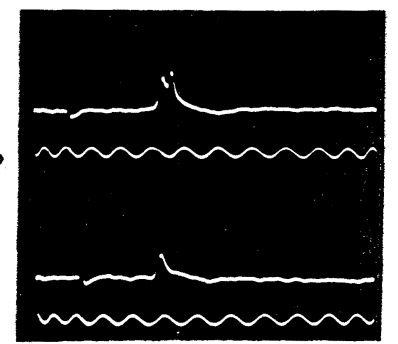

FIG. 10

FIG. 9. Temperature dependence of internodal inward currents. Ordinate, the current in percentage of the peak value of the outward current.

FIG. 10. Reduction in amplitude and duration of internodal inward currents due to inactivation of the distal node. $a$, the case of narcotization; $b$, the case of $40 \mathrm{mV}$ anodal polarization. In $a, 2,000 \mathrm{c} / \mathrm{s}$., $15 \mu$ thick fibre, $24.0^{\circ} \mathrm{C}$. In $b$, $1,000 \mathrm{c} / \mathrm{s} ., 14 \mu$ thick fibre, $13.0^{\circ} \mathrm{C}$. 
was about $23 \%$, the highest value obtained being $33 \%$ at $29.0^{\circ} \mathrm{C}$. Such a value seems to be too great to be assigned simply to a passive process.

Further, the amplitude gets reduced by about one-fourth, if the distal node is inactivated by narcotic or anodal polarization (fig. 10, $a$ and $b$ ), and this phenomenon too is favored by higher temperatures.

The duration behaves similarly, too.

\section{Inward current in successive impulse transmission}

Attempts were made to explore how the internode responds to successive transmission of impulses. An example is presented in fig. 11. It was confirmed that the inward component can follow each of the outward without any obvious change in amplitude, at least in the range of frequency smaller than 200 per sec.

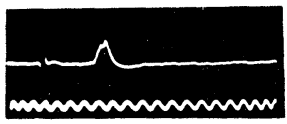

FIG. 11. Internodal membrane currents in successive impulse transmission. Top, taken as control with single stimulus; lower two, with successive stimulation of nerve trunk at intervals of 20 and $5 \mathrm{msec}$., respectively. 2,000 and $50 \mathrm{c} / \mathrm{s}$. $13 \mu$ thick fibre. $25.0^{\circ} \mathrm{C}$.

\section{CONSIDERATION}

1. The inward internodal membrane current accompanying conduction as an expression of activity. It was stated previously that the inward current accompanying conduction is an expression of an active but not of a simple passive process. It is, however, not without questions. First, the temperature dependence of the intensity (fig. 9) might be argued as due to change of resistance of the myelin sheath (Tasaki, 1955). But if so, then the magnitude relative to the outward current should be practically invariable regardless of temperature change. In fact, however, it was never so.

Next, the high threshold for evoking a response might appear to preclude the possibility for the internode to be activated by a usual nodal action current, and thus make the active nature of the inward component questionable. However, in normal conduction of nerve, the juxta-nodal portion of an internode will be supplied with almost the whole amount of the nodal action potential $(100-120 \mathrm{mV})$, which, if judged from our results, is never insufficient to elicit a larger or smaller response therefrom.

Further, the fact that the inward current was reduced in size and duration when the adjacent distal node was inactivated, is easily comprehensive as an expression of the graded character of the response, but not of a passive process. Thus, we are led to conclude that the inward internodal membrane current represents an active process, initiated by the nodal activity.

2. The high threshold in direct stimulation. The responses in question are 
characterized first of all by high threshold.

The high threshold must be correlated intimately with the high transverse resistance of the myelin sheath, which is, according to Tasaki's new measurements (1955), about $290 \mathrm{~m} \Omega$ for $1 \mathrm{~mm}$. length. Yet, it seems not sufficient for quantitative explanation of our results, for, the ratio of the resistance of a 0.8 $\mathrm{mm}$. long internode to that of a nodal membrane is about 9 , while the ratio of the rheobasic voltages actually found was about 15, which is inconformable with the resistance ratio. Furthermore, the threshold-reducing effects of the hypertonic solutions (Results, 2, $a$ ) is too great to be explained simply by their resistance-reducing action. These facts appear to be suggestive of some special character of the internodal excitable membrane, assignable presumably to the ionic milieu which may be different from that of the nodal membrane.

3. The graded nature. Another characteristic of the response is the graded nature of size and duration, a nature which characterizes several kinds of nerve and receptor cells, although somewhat different in details.

As stated before, the make response showed often a step-like rising phase (for weak stimuli), while the break response a short, spike-like configuration, although not always. The step-like shape might be due partly to some structural peculiarities as was supposed before, but if supposed further that there exists a certain "unitary" response in the spatial sense, then we can well understand the whole facts just mentioned, together with the fact of gradation observed.

Rushton (1937), later Yamagiwa (1955, 1957) postulated that a certain minimal length of fibre must be excited for initiation of impulse. According to Yamagiwa's estimation (p. 318, 1955), the minimal length for a nodal membrane occupies $0.05-0.5 \mu$, a fraction of a nodal breadth. We can suppose that the unitary response above suggested may be associated with such a spatial unit of membrane, and, on the other hand, that the internode studied (about $0.8 \mathrm{~mm}$. long) was wide enough to produce spatio-temporal summation of varying extent of such unitary responses, which is nothing but the so-called graded responses. If supposed further that repetition of local (non-conducting) activities of a certain number of such unitary spots takes place slightly asynchronously, then the make response of an internode as a whole can keep a plateau of a certain height during the whole period of stimulation, while the break response not.

4. Whether the internodal activity propagates or not. It seems certain now that the internode is excitable, but whether the activity spreads or not in the membrane is undecided. There are evidences showing the localization of activity, among others the fact that the internodal action current never appeared diphasic despite of some myelinated portions involved in the distal pool (figs. 1-6). This shows clearly the impossibility of conduction along or transmission over the air-gapped portion of the internode. And, if once the localization of activity is assumed, then it becomes easy to understand the graded nature of the response in a way as described above. However, there is no decisive positive evidence for the assumption, particularly in normal conditions. 
Another question is whether an internodal activity is capable of exciting the adjacent node. As far as examined in the experimental arrangement employed, there are evidences showing the disability, i.e., (1) the internodal responses were always monophasic as mentioned above (figs. 1-6), (2) an internodal activity did not affect that of the adjacent node (fig. 6), and (3) the intensity of an internodal action current seems to be insufficient for exciting a node, for, the largest amplitude of the inward internodal membrane current (that is, of the internodal action current) was $33 \%$ of the outward current at $29.0^{\circ} \mathrm{C}$., when caused by activities of two neighbouring nodes. This corresponds to $13 \%$ of the peak value of a nodal action current. Now, the safety factor for propagation in myelinated fibre is 5-7 (Tasaki, 1953) or 6-8 (Rosenblueth et al., 1949); a similar value (about 7) was found in the ratio of spike height to critical local response in good conditions (del Castillo and Stark, 1952). The value $13 \%$ may therefore be just about critical for activation of a node of Ranvier. If so, the intensity of the internodal action current must be below critical, if caused by a single active node as in normal conduction, because it will be reduced by about one-fourth as stated before (fig. 10). Thus, it seems certain that an internodal activity is incapable of exciting the adjacent node, at least in the experimental conditions given. We do not know, however, how the matter will be in normal conduction where, among others, no air-gap exists. We cannot say anything definite about it at present.

The final conclusions to be drawn after all, are the following two: (1) the internodal myelin-covered portion is not a simple physical cable, but a physiologically excitable structure which can be put into action by a nodal action current, and (2) there is no decisive evidence for whether the activity propagates or not. If it propagates, then the whole conduction will be continuous as was claimed by Rosenblueth et al. (1954). But if not, the conduction will be practically saltatory in the sense of Yamagiwa (1951), but not in the sense of Hodler et al. (1952) who take the internode inexcitable. The behavior of the internode in this case will be similar with that of the motoneurone soma or the spinal ganglion cell. They cannot be a mediator of an impulse: their spikes are set up later than the axon's and die out without playing any active role in the impulse transmission in normal state (cf. Eccles, 1957; Ito, 1957).

5. The responsible membrane. In the above considerations, there has been a tacit assumption that the excitable internodal membrane is a simple continuation of the nodal membrane. Actually, however, there is a set of membranes in the internode, corresponding to the numerous myelin lamellae. Each of these has been assumed to possess the qualification for a cell membrane in electrical properties (Hodgkin, 1951), but if they all should be excited, then a much stronger stimulus would have been needed than was found actually. The fact that the internode stands well the hypertonicity or narcotic action of the surrounding media, may be another indication for the responsibility of a certain inner, or presumably the innermost membrane, which is a direct continuation of the nodal membrane. 
SUMMARY

Evidences were presented showing that the internodes of myelinated fibres respond to externally applied outward current.

The responses are characterized by high threshold and graded nature in size.

Similar responses can be evoked from the juxta-nodal myelin-covered portions of a fibre.

Evidences were presented showing that the inward internodal membrane current accompanying normal conduction is an expression of the internodal activity.

It was concluded that the internode is excitable, but whether it propagates and whether it excites the adjacent node or not, are unknown.

Cordial thanks are due to Prof. K. Yamagiwa for his kind encouragement and pertinent criticism throughout this work, and to Prof. T. Wakabayashi and Prof. K. Matsuda for their valuable discussions.

\section{REFERENCES}

1. Del Castillo, J. And Stark, L. J. Physiol. 118:207-215, 1952.

2. ECCLES, J. C. The physiology of nerve cells. Baltimore: Johns Hopkins Press, 1957.

3. Hodgkin, A. L. Biol. Rev. $26: 339-409,1951$.

4. Hodler, J., Stämpfli, R. AND TASaki, I. Amer. J. Physiol. 170: 375-389, 1952.

5. HuXley, A. F. AND STÄmpfli, R. Arch. sci. physiol. 3: 435-447, 1949. (cited from Stämpfli, 1954).

6. ICHIOKA, M. AND Konishi, K. Jap. J. Physiol. $7:$ 12-19, 1957.

7. ITo, M. Jap. J. Physiol. $7:$ 297-323, 1957.

8. Konishi, K. Jap. J. Physiol. 7 : 1-11, 1957.

9. Maruhashi, J. ET AL. Jap. J. Physiol. 6 : 175-189, 1956.

10. Rosenblueth, A., Alanis, J. ANd Mandoki, J. J. cell. comp. Physiol. 33: 405-440, 1949.

11. Rosenblueth, A., Garcia Ramos, J. AND Miledi, R. J. cell. comp. Physiol. 43: 347364, 1954.

12. Rushton, W. A. H. Proc. Roy. Soc. B. 124 : 210-243, 1937.

13. SAto, M. AND Schneider, D. Z. Naturf. 9 b: 644-654, 1954.

14. StÄMPfli, R. Physiol. Rev. $34: 101-112,1954$.

15. TASAKI, I. Nervous Transmission, p. 44. Springfield, U.S.A.: Charles C. Thomas, 1953; Amer. J. Physiol. 181: 639-650, 1955.

16. TASAKI, I. AND Ushiy Ama, J. Arch. Inter. Stu. Neurol. 1: 1-5, 1950.

17. Yamagiwa, K. Jap. J. Physiol. 2: 79-92, 1951; 5: 131-159, 317-321, 1955; Tohoku J. exper. Med. 66 : 153-185, 1957. 